\title{
Contextualización, usos empíricos y etnografía de las redes sociales en el Ciberperiodismo
}

\author{
Jesús Miguel FLORES VIVAR \\ Universidad Complutense de Madrid \\ jmflores@ucm.es
}

Recibido: 16 de julio de 2015

Aceptado: 18 de septiembre de 2015

\begin{abstract}
Resumen
Las redes sociales en Internet son un fenómeno de masas cada vez más importante en el ecosistema de medios digitales, tanto así que ya no se concibe un artículo que no tenga implementado una red social. De ahí que el público usuario de las redes sociales es, en muchos casos, parte de la audiencia de los medios periodísticos. Pero, para conocer en profundidad esa parte de la audiencia digital que usan las redes sociales y el impacto que hacen en el entorno de los medios de comunicación es importante estudiar quienes están detrás de las comunidades de redes sociales virtuales. Pero, estos usuarios ¿Qué hacen?, ¿Que piensan?, ¿Cuál es su opinión respecto a una noticia?, ¿Qué necesidades tienen? ¿Qué tipo, modelo y forma de ver la información desean? En este contexto, la investigación etnográfica y antropológica con todas sus técnicas juega un papel fundamental en todo estudio de este tipo. Pero ¿En que medida la etnografía y la antropología pueden ayudar a ser más eficaces con los mensajes del ciberperiodismo? ¿Nos ayuda la etnografía a conocer el público que está detrás de las redes sociales que acceden a los artículos u otras informaciones de los medios? ¿Es importante conocer los usos y costumbres de los usuarios de las redes sociales? ¿Deben los medios adaptarse a los nuevos entornos etnográficos virtuales de donde emerge un nuevo periodismo basado en la participación? El presente artículo intenta responder a estas y otras variables.
\end{abstract}

Palabras clave: redes sociales, internet, etnografía, comunicación, ciberperiodismo.

\section{Contextualization, Uses Empirical and Ethnography of Social Networks in the Cyberjournalism}

\begin{abstract}
Social networking sites are a phenomenon of masses increasingly important in the digital media ecosystem, and not so much news that does not have implemented a social network with links. Hence, the public user of social networks is, in many cases, part of the audience of the media. But to know in depth this part of the digital audience using social networks and the impact they make on the environment of the media is important to study those behind the virtual social networking communities. But these users, what do they think? What is their opinion on a news?, What are their needs? What type, model and way to see the information they want? In this context, the ethnographic and anthropological research with all its technical to play a key role in any study of this type. But, what extent ethnography and anthropology can help they be more effective with messages of online journalism? Does it help to know ethnography public behind social networks accessing articles or other media reports? Is it important to know the customs of the users of social networks? Should the means to adapt to new environments where virtual ethnographic emerges a new journalism based on participation? This article attempts to answer these and other variables.
\end{abstract}

Keywords: social network, internet, cyberjournalism, ethnography, communication.

\section{Referencia normalizada}

FLORES VIVAR, Jesús (2015): “Contextualización, usos empíricos y etnografía de las redes sociales en el Ciberperiodismo". Estudios sobre el Mensaje Periodístico. Núm. especial diciembre, págs.: 81-95. Madrid, Servicio de Publicaciones de la Universidad Complutense.

Sumario: 1. Introducción. 2. Hipótesis y objetivos. 3. Fuentes y metodología. 4. Las redes sociales desde una perspectiva antropológica y etnográfica. 5. Discusiones: avances y propuestas del método etnográfico en el análisis de medios sociales en Internet. 6. Conclusiones. 7. Referencias bibliográficas. 


\section{Introducción}

Estudiar el periodismo en el ciberespacio (utilizando los diferentes recursos sociales de Internet), que ha derivado en el emergente concepto del ciberperiodismo, nos reconduce a adoptar nuevos aspectos de la investigación social en esta disciplina del conocimiento que es el pilar de toda democracia. Así, el objetivo fundamental es ayudar a la pervivencia del periodismo de calidad en los nuevos entornos digitales y bajo los recursos que proporciona internet como son los medios y redes sociales.

Las redes sociales en Internet han dejado de ser un paradigma comunicacional para convertirse en elementos clave que configura nuevos procesos de comunicación, hecho que cambia teorías las cuales hemos desarrollado durante décadas. Hemos pasado del concepto de fines del milenio "si no estás en internet, no existes" a la situación actual de "si no estás en las redes sociales, no existes".

En este contexto, el paradigma actual es:

"generar una cultura de red. Internet se ha convertido en la plataforma que almacena un cúmulo de conocimiento, derivada de la gran cantidad de investigaciones e innovaciones elaboradas por el talento, la imaginación, la audacia y la inteligencia de los usuarios de la Red. En coincidencia con expertos, la eclosión de Internet nos ha llevado a ver sorprendentes experiencias en red, diseñadas, realizadas y ejecutadas por ciudadanos de a pie, en la gran mayoría sin mayor formación y en lo que se ha venido a denominar la ciencia de las redes o cultura de red" (Flores, 2009: 75).

Pero, las redes sociales en Internet (que denominaremos en adelante, redes sociales) poseen estructuras y se rigen por patrones, cuyas características son la interactividad y la interacción entre personas vinculadas en función a intereses comunes o como nodos familiares. El periodismo y los medios, desde hace algunos años han incorporado características propias de las redes sociales en la creación de contenidos. Actualmente, no existe un medio o un artículo periodístico en donde las redes sociales no estén presentes. Pero ¿basta esta alternativa de difusión para señalar el acierto del medio? ¿Acrecentará esta acción la pervivencia y crecimiento del medio? A primera vista las redes aparecen como un entorno complejo difícil de desentrañar. Sin embargo, detrás de esa maraña existen patrones de interacción, que influyen en el comportamiento de las personas u organizaciones implicadas, aunque no sean conscientes de esta influencia (Molina y Ávila, 2002). Desde esta perspectiva, la sociedad no la conforman los individuos, sino un conjunto de individuos regidos por determinados patrones de relaciones, simultáneamente. Y los estrategas de los medios en materia de redes sociales no terminan de darse cuenta de ello.

Por otra parte, los usuarios de redes sociales en Internet dan sentido a sus prácticas a través de una comprensión compartida, que surge tanto de la producción de una página web como del uso de un grupo de noticias, y que constituyen nada menos que formas de acción social (Hine, 2004). Una evolución natural ha sido la creación de grupos de noticias, que no son más que espacios sociales altamente diferenciados sostenidos por colaboraciones de usuarios en forma de notas y mensajes temporales y situacionalmente relevantes para una comunidad en Internet. Más que trascender el tiempo y el espacio, las redes sociales en Internet puede ser representada como una instancia de múltiples órdenes espaciales y temporales que cruzan una y otras vez la línea divisoria entre lo online y lo offline. 
En la dicotomía periodismo y medios sociales, se vienen abordando investigaciones cada vez más profundas y exhaustivas y desde diferentes ángulos. Todo esto porque el foco esta puesto en la pervivencia del periodismo y, por consiguiente, de los medios como empresas informativas en donde ya es innegable el uso de los recursos como las redes sociales o las funcionalidades que tienen los medios sociales como los blogs.

En este contexto, la pregunta que surge es: ¿Qué investigamos de las redes sociales? ¿Interesa investigar el software o la interfaz de la red social? O, por el contrario ¿interesa investigar el uso que se da a través de esas interfaces? Considerando que son individuos o conjuntos de estos los que están detrás de esas interfaces de redes sociales ¿es interesante para el medio que es lo que hace interactuar al usuario de cara a un mensaje o artículo periodístico? ¿Cuáles son sus necesidades? ¿Qué opinan sobre tal o cual tema?

Para responder a estas variables se han abordado investigaciones desde perspectivas más sociológicas y económicas. Pero, se ha dejado de lado el estudio de los usuarios desde su perspectiva más antropológica y etnográfica. De hecho, cada vez más investigaciones sobre Internet y redes sociales se está constituyendo en un ámbito de enorme interés etnográfico y antropológico (Contreras, 2004, 53). ¿Y porque ahora el interés por este entorno? La respuesta es simple pero contundente: porque necesitamos saber cuáles son los usos, costumbres, deseos, necesidades, creación, opiniones, contextualización, etc., de los usuarios que, formando comunidades virtuales, intercambian mensajes, también, a través de plataformas virtuales. Por tanto, es probable que este sea el elemento importante en muchos estudios e investigaciones sobre redes sociales y su relación con el periodismo y los medios. No se han tenido en cuenta investigaciones en donde la etnografía juega un papel fundamental en este estadio de la red y sus implicaciones en una sociedad cada vez más interconectada.

Para Robert A. Hanneman (2000), no hay nada inusual en los datos utilizados en el análisis de redes sociales. Es cierto que quienes trabajan con redes utilizan un lenguaje especializado para describir la estructura y los contenidos de los conjuntos de observaciones estudiados, pero los datos de redes también pueden describirse y entenderse utilizando las perspectivas y los conceptos propios de métodos más familiares como la investigación sociológica tradicional (cross-sectional survey research). En esta línea, matiza:

"Sin embargo, a menudo los datos que los investigadores en redes sociales estudian resultan muy distintos de la tradicional matriz rectangular tan familiar para los investigadores y el análisis estadístico. Las diferencias son importantes porque nos llevan a observar los datos de una manera distinta -y también a pensar de manera distinta- sobre cómo aplicar los procedimientos estadísticos. Los datos sociológicos "convencionales" están compuestos por una matriz rectangular con mediciones. Las filas de la matriz son los casos, sujetos u observaciones. Las columnas son las puntuaciones (cuantitativas o cualitativas) de los atributos, variables o mediciones. Entonces, cada celda de la matriz describe la puntuación de algún actor con respecto a algún atributo. En algunos casos, estas matrices pueden tener una tercera dimensión que representa los cuadros de observaciones de múltiples grupos" (Hanneman, 2000). 
El periodismo, como expresión cultural y evolución del ser humano se nutre directamente de un formato de características que llamamos Técnicas Etnográficas. También las tecnologías de la información como factor de producción de los individuos que repercuten en estilo de vida son analizadas por dichas técnicas. En este sentido, si juntamos las tecnologías digitales, abanderadas por Internet, como expresión de producción y el periodismo como expresión cultural, encontraremos nuevos escenarios en donde la etnografía y la antropología juegan un papel fundamental en el intento por hacernos entender el nuevo espacio en lo que se ha venido a denominar cibercultura.

Desde hace pocos años, han emergido algunos autores quienes definen que el interés por la etnografía virtual, o en línea, surge cuando el objetivo no es sólo estudiar los usos de Internet, sino también las prácticas sociales en línea y de qué manera estás prácticas son significativas para las personas y cambian estilos y formas de comunicar. En este contexto, la configuración de múltiples comunidades virtuales generan organismos independientes que se constituyen con reglas propias e intereses comunes. De este modo, la Red se constituye en una instancia de desempeño general donde los usuarios construyen formas comunes y determinadas de actuar, haciendo las veces de estabilizadores de usos de Internet. Las relaciones sociales que se forman en Internet también tienden a estabilizar la tecnología, así como a fomentar la comprensión común de sus formas específicas de ser y funcionar.

Obviamente, atendiendo a las características de las redes sociales, los medios de comunicación han incorporado y adoptado estas tecnologías de participación como una estrategia para viralizar sus noticias, dado que el modelo de negocio de los medios online, se mide, actualmente, por la cantidad de accesos que tiene un determinado sitio web. Estas son perspectivas que pretenden investigar el camino de interacción y consolidación de socialización en el ciberespacio y la posible existencia de conexiones (o no conexiones) entre usuarios de redes sociales de los medios.

El artículo, desde una perspectiva etnográfica, aborda las múltiples formas de expresión del comportamiento social en los espacios de socialización que integran una comunidad de redes sociales virtuales utilizadas en el contexto de los medios españoles.

El presente trabajo, forma parte de la tercera y última fase de la investigación sobre periodismo y redes sociales que se desarrolla dentro del proyecto competitivo "Cybermedia II: Desarrollos e innovaciones del periodismo en las redes sociales en Internet y telefonía móvil. Convergencias, modelos de negocios, servicio y formación"”.

\section{Hipótesis y objetivos}

Es ampliamente sostenido que los estudios de ciencia y tecnología han alterado radicalmente los enfoques pasados sobre la tecnología, desplazando así la concepción lineal del cambio tecnológico y posibilitando la apertura de sólidos programas de investigación que están dando como resultado una verdadera renovación teórica (Es-

${ }^{1}$ MINECO -Plan Nacional de I+D+i- Referencia: CSO2011-25235. 
cobar, 2005:16). Esta renovación teórica hace que los procesos de comunicación que se dan a través de las redes sociales se hayan transformado radicalmente.

Las TIC, abanderadas por Internet, han facilitado la creación de nuevos escenarios comunicativos e interactivos. Estos espacios han ido evolucionando y adaptando características de anteriores espacios con el objetivo de ofrecer una experiencia renovada. En este contexto, en 2004 aparece Facebook y en 2006, Twitter, redes sociales que se han convertido en el paradigma de la Web 2.0, y al mismo tiempo, en dos de las principales plataformas virtuales para la comunicación mediada por ordenador. Facebook y Twitter, son en la actualidad, las redes sociales más utilizadas, con más de mil millones de usuarios activos la primera y más de 700 millones de usuarios la segunda. En noviembre de 2014, según la web Worldometers, el 8,4\% de la población mundial era usuaria activa de Facebook.

Entendido el alcance de estos espacios de interacción y comunicativos, es de especial interés para la Etnografía y Antropología comprender el uso que los individuos dan a estas redes sociales. Así, según la descripción de Sören Romero (2014) para la red social Facebook, el acercamiento a las redes sociales por parte de la Etnografía Virtual se puede hacer desde distintos enfoques. Parte de estos enfoques se han constituido en características esenciales en el estudio de las comunidades de usuarios de redes sociales en Internet que forman parte de la audiencia digital de los medios online. Estas características son:

1. Como herramienta de investigación. Las redes sociales se han convertido en una potente herramienta de investigación. Su tasa de penetración a casi todos los niveles demográficos permite investigar casi cualquier tema en comunidades virtuales en torno a una misma temática. Además, permite clasificar las opiniones a través de variables de interés para el/la investigador/a, como puede ser la composición de la foto de perfil o los datos facilitados por el/la usuario/a para la construcción de la identidad en estas redes sociales.

2. Como fuente de datos. Las redes sociales permiten acceder a una ingente cantidad de datos de individuos y sus interacciones, al quedar registradas y poder ser revisadas por el/la etnógrafo/a tiempo después. Las acciones que pueden llevar a cabo los/as usuarios/as, tanto de Facebook con "Me gusta" como de Twitter con el "retuit" a una publicación, "compartirla" o comentarla ofrece información de carácter cultural muy valiosa y con múltiples formatos.

3. Como contexto donde ocurre la acción. En la actualidad, las redes sociales se han posicionado como un espacio comunicativo de preferencia por la mayoría de los usuarios de Internet y como tal es el lugar en donde se llevan a cabo interacciones que son de interés para la etnografía virtual. Estas interacciones pueden ir desde la simpleza de las narraciones que hacen los usuarios sobre eventos comunes del día a día, hasta interacciones más elaboradas en torno, por ejemplo, a temas políticos, sociales o económicos.

4. Como plataforma de negocios y emprendimiento. Los usuarios de redes sociales dan rienda suelta a sus opiniones basándose en el anonimato (o no) sobre distintos temas (noticias) que se tratan en los medios informativos. Esta acción produce una viralización de los contenidos periodísticos, lo que se traduce en una cantidad 
creciente de accesos a los sitios periodísticos producido por los enlaces que los usuarios colocan en sus "muros" o en sus "tuits", lo que repercute en el modelo de negocio de los medios. Al mismo tiempo, existen cada vez más casos en los que las plataformas de redes sociales se han convertido en generadores de negocio por parte de emprendedores que han sabido utilizar estas tecnologías con bastante acierto.

Según Escobar (2005: 9) como un nuevo campo de práctica antropológica, el estudio de la cibercultura está relacionado particularmente con las construcciones y reconstrucciones culturales en las que las nuevas tecnologías están basadas y a las que a su vez ayudan a tomar forma. En este contexto y tomando como referencia dicha afirmación, el punto de partida de este cuestionamiento es la creencia de que las redes sociales representan una invención cultural en el sentido de que ésta contribuye a formar un mundo nuevo. Distintos investigadores antropológicos coinciden en que toda tecnología emerge de unas condiciones culturales particulares y de forma concomitante ayuda a producir otras. Los antropólogos podrían llegar a estar bien preparados para entender estos procesos si están abiertos a la idea de que la ciencia y la tecnología son campos cruciales para la creación cultural en el mundo contemporáneo.

Por otra parte, es ampliamente sostenido que los estudios de ciencia y tecnología han alterado radicalmente los enfoques pasados sobre la tecnología, desplazando así la concepción lineal del cambio tecnológico y posibilitando la apertura de sólidos programas de investigación que están dando como resultado una verdadera renovación teórica (Escobar, 2005). En una línea similar, distintos investigadores de las ciencias sociales no dudan en afirmar que atravesamos una etapa en la cual, las investigaciones en el ámbito del periodismo y los medios de comunicación requieren de técnicas que se utilizan en la ciencia de la antropología y la etnografía.

Para Cristine Hine (2004), hasta ahora, "se ha dedicado mucho más esfuerzo a predecir un futuro revolucionario de Internet que a investigar, en detalle, cómo se utiliza y de qué modos se incorpora a la vida cotidiana de las personas". Creemos pues, que ha llegado el momento de dedicar los esfuerzos a investigar estos aspectos que son cruciales para el periodismo y los medios digitales. Lo que implica tener que potenciar la investigación y análisis de las redes sociales pero desde una perspectiva más social, mas antropológica, estudiando los usos, costumbres, deseos, modos de vida, socialización, etc., situación que permitiría intentar encontrar la piedra filosofal de la rentabilidad económica para el periodismo y los medios. De ahí que resulta interesante aplicar el método abordado por Hine (2004) explorando una metodología de investigación sobre internet para el estudio empírico sobre los usos actuales de las redes sociales.

Hine (2004) sostiene que el agente de cambio no es la tecnología en sí misma, sino los usos y la construcción de sentido alrededor de ella, razón por la cual existe un espacio de estudio sobre las prácticas cotidianas en torno a Internet. Y en nuestra opinión, en todo lo que de la red se deriva: el ciberperiodismo y los medios sociales. La etnografía es una metodología para iniciar este tipo de estudios, en la medida en que puede servir para explorar las complejas interrelaciones existentes entre las aserciones que se vaticinan sobre las nuevas tecnologías en diferentes contextos: en el hogar, en el trabajo y por consiguiente, en los medios de comunicación y en las revistas y publicaciones académicas: 
"Una etnografía de Internet puede observar con detalle las formas en que se experimenta el uso de una tecnología. En su forma básica, la etnografía consiste en que un investigador se sumerja en el mundo que estudia por un tiempo determinado y tome en cuenta las relaciones, actividades y significaciones que se forjan entre quienes participan en los procesos sociales de ese mundo. El objetivo es hacer explícitas ciertas formas de construir sentido de las personas, que suelen ser tácitas o que se dan por supuestas. El etnógrafo habita en una suerte de mundo intermedio, siendo simultáneamente un extraño y un nativo. Ha de acercarse suficientemente a la cultura que estudia como para entender cómo funciona, sin dejar de mantener la distancia necesaria para dar cuenta de ella" (Hine, 2004)

En este sentido, consideramos que la perspectiva etnográfica puede adaptarse para la comprensión sobre cómo se constituye las redes sociales a través de su uso en el contexto actual. Así, esta metodología nos permite centrarnos en lo que Knorr-Cettina (1983) llama "el carácter ocasionado, localmente situado" del uso de Internet y específicamente de las redes sociales. Por tanto, el objetivo será estudiar cómo se negocia el estatus de las redes sociales en el contexto del ciberperiodismo y los medios en Internet.

Según Hine (2004) las creencias acerca de Internet pueden tener consecuencias importantes sobre la relación individual que se tenga con la tecnología y sobre las relaciones sociales que se construyan a través de ella. La etnografía, en este orden de cosas, puede servir para alcanzar un sentido enriquecido de los significados que va adquiriendo la tecnología en las culturas que la alojan o que se conforman gracias a ella. Y las redes sociales son manifestaciones de esas culturas alojadas en las plataformas de internet.

En esta línea, el estudio abordado se inicia dialogando con las proyecciones teóricas acerca del significado de Internet y las plataformas de redes sociales, utilizándolas para resolver los problemas que plantearía una etnografía del uso de los medios sociales. Algunas interrogantes que se desprenden a partir de nuestra revisión teórica y que pretendemos explorar, son:

- ¿Cómo los usuarios llegan a comprender las capacidades y posibilidades de Internet y las redes sociales? ¿Qué implicaciones tiene su uso? ¿Qué interpretan de ella en tanto medio de comunicación y a quién perciben como audiencia?

- ¿De qué modo afectan las redes sociales a la organización de las relaciones sociales en el tiempo y el espacio? ¿Es distinta esa organización a la de "la vida real"? Y si la respuesta es afirmativa, ¿cómo los usuarios reconcilian lo virtual y lo real?

- ¿Cuáles son las consecuencias de las redes sociales sobre los sentidos de autenticidad y autoría? ¿Cómo se desempeñan y experimentan las identidades, y cómo se juzga la autenticidad?

- ¿Es "lo virtual” experimentado como algo radicalmente diferente y separado de "lo real”? ¿Hay una frontera divisoria entre la vida online y offline?

\section{Fuentes y metodología}

Con los antecedentes descritos, una línea de investigación que abre diversos campos de análisis en la comunicación mediada por ordenador se produce a través de la etnogra- 
fía aplicada a la virtualización de las redes sociales y su impacto en el periodismo online o ciberperiodismo. Según Etchevers (2010), la respuesta se da mediante un análisis cualitativo de la presencia y formas de comunicación de los usuarios de medios sociales en Internet, en situaciones de relación interpersonal o de comunidades online.

En este contexto, nuestro objeto de estudio son las redes sociales en Internet como parte de la audiencia de los medios periodísticos digitales. Y considerando que Internet representa un lugar donde se gesta una cultura, el ciberespacio, establecemos allí nuestro punto de partida. El segundo componente del estudio es la comunicación mediada por ordenador (CMC Computer-Mediated Communicactions) lo que nos lleva a realizar un esfuerzo experimental, intentando entender los hábitos y costumbres de las personas usuarias de redes sociales. Y es que algunos expertos coinciden en afirmar que este medio, CMC, empobrece la comunicación, dado que las personas ven limitada su capacidad de expresión a comunicaciones textuales, y cuando la transmisión de códigos sociales vitales se restringe, se generan agresiones y malos entendidos. En contrapartida a este pensamiento, surgen investigadores, como Rheingold (1993) que no dudan en afirmar que las CMC proveen formas de interacción muy ricas, así como de proporcionar el espacio idóneo para la conformación de comunidades en internet.

Según Hine (2004), las interacciones cara a cara, así como las retóricas sobre viajar a sitios remotos, han sido determinantes para dar autenticidad a las descripciones etnográficas. Es cierto que un medio limitado como las CMC podría hacer tambalear algunas asunciones centrales de la etnografía, tales como que el conocimiento se ha de evaluar a través de la experiencia y la interacción; pero, "el cuadro cambia de algún modo si simplemente reconocemos que el etnógrafo puede construirse a sí mismo como un actor que necesita tener experiencias similares a las de sus informantes".

Realizar una investigación etnográfica a través de la $\mathrm{CMC}$ abre la posibilidad de enriquecer las reflexiones acerca de lo que significa ser parte de las redes sociales en Internet y cómo los medios de comunicación pueden perfeccionar sus estrategias de fidelización de la audiencia digital. Y las técnicas a utilizar en la investigación de redes sociales en Internet son las que proceden del campo de la antropología, derivados en métodos etnográficos como la observación participante, el uso de fuentes primarias y las entrevistas sumadas a las encuestas.

La observación y el conocimiento confirman que siempre estamos comunicando y siempre hay emociones involucradas (Etchevers, 2011). Estas emociones se repiten en la comunicación e interacción que hacen los usuarios de redes sociales en Internet. Sin embargo, diversos factores implican la necesidad de demostrar esta afirmación e implementarla en ámbitos del periodismo y los medios en Internet. La investigación realizada en este estudio pretende demostrar que es posible una comunicación eficaz a través de interfaces virtuales, estudiando las emociones, las capacidades y habilidades, necesidades, deseos y opiniones de los sujetos que están detrás de esas interfaces virtuales. Conocer en detalle los nuevos usos y costumbres de la personas usarías de redes es el lei motiv de los medios, empecinados en encontrar el modelo de negocio en entornos del ciberespacio.

Para llevar a cabo esta metodología cualitativa, se consideró la alternativa etnográfica por ser un método de trabajo que describe directamente las múltiples formas 
de vida de los seres humanos, mediante la observación, participación y descripción comprensiva de lo que la gente hace, cómo se comporta y cómo interactúan entre sí. Específicamente, una etnografía en Internet ayuda a entender la forma en que se gesta la interacción y se experimenta el uso de una tecnología determinada en dicho proceso. Planteado así, adentrándose en el ciberespacio y formando parte de éste, se puede comprender mejor cómo se transmiten las emociones en la comunicación textual mediada por ordenador.

Según Hine (2004: 18) los primeros estudios sobre comunicación mediada por ordenador ( $\mathrm{CMO}$ o $\mathrm{CMC}$ en inglés), realizados con un fuerte acento experimental, concluían que este medio empobrecía la comunicación (por ejemplo, cuando las personas ven limitada su capacidad de expresión a comunicaciones textuales, y cuando la transmisión de códigos sociales vitales para la comunicación se restringe, se generan agresiones y malentendidos). Sin embargo, también afirma que esté en total desacuerdo con esa afirmación puesto que no considera que la limitación de este medio genere necesariamente agresiones y malentendidos. Partiendo de estas investigaciones y coincidiendo con lo aportado por Hine, se hace necesario diseñar una investigación que demuestre que la comunicación textual mediada por ordenador (CMC) es de vital importancia para el buen entendimiento de las personas y que, efectivamente, las emociones son transmisibles por este medio.

Se debe tener en cuenta que la metodología etnográfica no queda ajena al contexto en que se desarrolla y por eso se considera desde una perspectiva adaptativa que reflexiona precisamente alrededor del método. De esta forma, en la medida que el investigador avance, conozca y experimente su problema de estudio, surgen nuevas opciones de investigación que le descubren opciones que en un principio puede no haber tenido previstas (Hine, 2004: 35-36). La investigación en redes sociales en el ciberespacio y con un enfoque a/de los medios de comunicación en Internet es descubrimiento y reflexión continuos. El investigador que utiliza las técnicas etnográficas, en lugar de permanecer aislado o ausente, se hace visible y activo en el contexto del trabajo de campo, haciendo una inmersión en las comunidades de redes sociales puede encontrarse con nuevas preguntas, probar o refinar nuevos conceptos analíticos lo cual, sea dicho de paso, permite tener una constante reflexión acerca de lo que significa ser usuario de las comunicaciones mediadas por ordenador.

Para Nadruz (2010) abordar un estudio etnográfico de las redes sociales virtuales, requiere de una labor metodológica aplicada específicamente para una etnografía online, por lo que se hace necesario configurar la investigación en tres etapas diferentes y correlativas, con motivo de permitir un alcance y precisión más clara y concisa a los objetivos trazados enfocándose desde lo general a lo particular, desde lo diferido a lo directo, desde lo abarcado a lo puntual. Así, las técnicas etnográficas para este tipo de estudio pueden ser 1) Observación flotante, 2) Observación diferida y 3) Entrevista semi-estructurada online a través de un canal de chat.

Con estas técnicas, vemos que la investigación sobre redes sociales en espacios virtuales debe basarse en una metodología cualitativa que presente su fundamentación en el trabajo de campo desarrollado, en el cual se ha logrado un acervo de evidencia documental y entrevistas mediante la observación participante en el contexto. 


\section{Las redes sociales desde una perspectiva antropológica y etnográfica}

Etnografía significa literalmente: descripción de un pueblo. Por tanto, la etnografía se ocupa de las personas en sentido colectivo, no de los individuos. Así, es una manera de estudiar a las personas en grupos organizados duraderos a los que cabe referirse como comunidades o sociedades (Angrossino, 2012:19). La etnografía es ante todo una actividad investigativa de corte descriptivo que pretende estudiar la cultura de una comunidad. Etimológicamente está formada por dos vocablos: "graphos", que significa yo escribo y "Ethnos", que significa tribu o pueblo, es decir la etnografía apuntaría a la descripción de los pueblos, de su cultura de sus usos y costumbres.

Estudiar comunidades es el objeto de este trabajo. Pero, es un tipo de comunidades propias y emergentes de este milenio, que se desarrollan a través de interfaces de comunicación mediadas por ordenador (Computer-Mediated CommmunicationsCMC), conocidas como Comunidades virtuales. Joseph Dumit (2014: 350) habla de Implosión de Proyectos, que define como los intentos de enseñar y aprender acerca de la incrustación de objetos, hechos, acciones y personas en el mundo y el mundo en ellos. En nuestro caso, el de las redes sociales. El énfasis está en los detalles y las conexiones no obvias, así como sobre las múltiples dimensiones con el que podemos analizarlas: laborales, profesionales, materiales, tecnológicos, políticos, económicos, simbólicos, textual, corporales, histórico, educativo.

Desde una perspectiva antropológica es difícil encontrar una corriente teórica dentro de la cual situar este tipo de estudio. Contreras (2004:54) afirma que si tuviera que elegir alguna etiqueta de manera obligatoria para un proyecto de investigación de esta naturaleza lo denominaría "Antropología o etnografía del Ciberespacio". En una línea similar, Escobar (2005) afirma que actualmente están tomando lugar cambios considerables tanto en el carácter de la tecnología como en la manera en que la entendemos.

"La información computarizada y las biotecnologías están produciendo una transformación fundamental en la estructura y en el significado de la cultura y de la sociedad moderna. Dicha transformación no sólo es susceptible al cuestionamiento de la antropología, sino que quizás constituye un campo privilegiado para avanzar en el proyecto antropológico de comprender las sociedades humanas desde los puntos de vista estratégicos de la biología, el lenguaje, la historia y la cultura. En este artículo reviso las clases de análisis que están siendo desarrollados sobre la naturaleza social, el impacto y el uso de nuevas tecnologías; además sugiero contextos y pasos adicionales para la articulación de una "antropología de la cibercultura" (Escobar, 2005)

Las sociedades modernas son, al mismo tiempo, sociedades globalizadas en donde las fronteras se están diluyendo. Y más en el mundo virtual, en donde las personas de una comunidad no se identifican por la territorialidad, sino por los intereses y afinidades. Para Marc Auge (2007:17) "Si pensar en el concepto de frontera resulta útil es porque constituye el centro de la actividad simbólica que -según las teorías de LéviStrauss- se ha utilizado desde la aparición del lenguaje para dar un significado al universo y un sentido al mundo, a fin de que sea posible vivir en ellos". Es evidente que en la época actual en el que nos encontramos, para la aplicación de los estudios antropológicos y etnográficos en donde los conceptos de globalización y tecnologías están estrechamente relacionados, hablar de fronteras resulta un tanto utópico. 
Pero ¿Qué son las redes sociales? Es necesario aclarar en primer lugar que se trata de un concepto polisémico. Según José Luis Molina y Javier Ávila (2002), un primer sentido se refiere a las redes sociales como "networking" entre personas mediante plataformas virtuales. Este sentido ha desplazado, por su reciente popularidad, a otros con mucha más tradición en las ciencias sociales. Un segundo sentido se refiere a las redes sociales como metáforas sobre la complejidad del mundo social. Un tercer sentido se refiere a las redes como estrategia de "empoderamiento" de actores locales (redes de organizaciones normalmente) para la mejora de una situación o la puesta en marcha de una iniciativa. Finalmente, un cuarto sentido se refiere a las redes sociales como herramienta para el análisis de las relaciones empíricas entre personas, organizaciones, países u otras unidades de análisis.

Pero, el foco de nuestro análisis se centra en las redes sociales que fagocitan el crecimiento de los medios de comunicación en Internet. Considerando que las redes están conformadas por grupos de individuos, es necesario destacar cómo planteamos una investigación sobre comunidades de redes sociales en Internet, combinando otras disciplinas del conocimiento como la antropología y la etnografía y sus técnicas de investigación.

\section{Discusiones: avances y propuestas del método etnográfico en el análisis de me- dios sociales en Internet}

El estudio de las redes sociales en Internet se inscribiría en el contexto de la denominada "antropología o etnografía del ciberespacio". Aunque, para autores como Contreras (2004), quizás sea más apropiado hablar de antropología o etnografía en el ciberespacio, puesto que las practicas objeto del estudio se desarrollan casi íntegramente en la Red. No obstante, los temas a través de las interacciones que se dan, fundamentalmente, a través de las redes sociales, forman parte de categorías mucho más generales, como los movimientos sociales o la construcción de conocimiento, que se extiende más allá del ciberespacio, "hasta los territorios que han sido los tradicionales objetos de estudio de la disciplina antropológica".

Aunque resulte una obviedad, estudiar a las comunidades online que se generan a través de las plataformas y usuarios de redes sociales, es hacer referencia obligada al estudio de las particularidades del espacio virtual. Afortunadamente, hay algunos pioneros en propuestas metodológicas, cuyos planteamientos ayudan a definir la aproximación y las técnicas que se deben usar en proyectos de investigación sobre redes sociales y la construcción del conocimiento para fortalecimiento del ciberperiodismo (o periodismo en la Red). Estudios como el de Meyer (1996) o Paccagnella (1999) citados por Contreras (2004:56), aportan pistas interesantes respecto a la observación en entornos virtuales y la validez de los datos así recogidos. Por su parte Hine (2004), agrega que Internet, permite un estudio detallado de las relaciones en línea, de modo que la red no es sólo un medio de comunicación, sino también un artefacto cotidiano en la vida de las personas y un lugar de encuentro que permite la formación de comunidades, de grupos más o menos estables, y, en definitiva, la emergencia de una nueva forma de sociedad o sociedad red, es la que debemos estudiar en profundidad. Es la nueva audiencia de los cibermedios, cuya característica en el ciberespacio es la 
participación activa, influenciada por los modos, usos y costumbres de la vida real que traslada al espacio virtual lo que convierte a las redes sociales en "plazas virtuales" donde se comenta, se habla, se conversa desde temas científicos, políticos, económicos y sociales hasta lo más baladí, exactamente igual como se hace en las plazas reales. Para Molina y Ávila (2002), la experiencia nos enseña que las dificultades para el análisis de redes sociales residen, más que en cuestiones conceptuales o teóricas, en el aprendizaje de la mecánica de la transformación de datos.

Así pues, dado que en los trabajos de los teóricos nombrados se proponen metodologías que a juicio de cada uno/a pueden ser las más apropiadas, hemos considerado que para el estudio de las redes sociales en Internet, concretamente, para las comunidades de usuarios de redes sociales que, directa o indirectamente, participan en la consolidación del ciberperiodismo, proponemos las siguientes técnicas de investigación etnográfica, tanto cualitativas como cuantitativas.

Las técnicas cualitativas se basan en la identificación-interpretación del objeto de estudio y son:

\section{Observación participante en las distintas plataformas de redes sociales}

La observación es el acto de percibir las actividades e interrelaciones de las personas en el entorno de campo mediante los cinco sentidos de la investigación (Angrosino, 2012: 61). Por tanto, no cabe duda que una parte importante de las investigaciones se dan a través de este método, dado que permite al investigador hacer una inmersión en el objeto de estudio a través de la observación directa y participante. Para ello, el/la investigador/a debe establecer contacto con las comunidades de redes sociales, previo registro de la plataforma elegida. En esta fase, en la que se asigna un cronograma de duración en el espacio-tiempo, se establece contacto directo con los usuarios, por ejemplo, de un medio o periódico, a través de mensajes que pueden etiquetarse, acción que redunda en un conocimiento de ciertas características de los usuarios de las redes sociales por las afinidades o diferencias que se pueden descubrir. En muchos casos, la observación participante permite entender el contexto de las interacciones de las comunidades online así como la comprobación de la hipótesis del proyecto en cuanto a referencia de usos y costumbres de los usuarios de redes sociales.

\section{Entrevistas mediadas por ordenador (o por teléfono) y de forma presencial con la muestra de la población seleccionada}

La entrevista es un proceso por el que se dirige una conversación para recoger información (Angrosino, 2012: 66). Para los casos de investigación del periodismo relacionada con las redes sociales, una vez hecha la selección de las personas a entrevistar (tanto de usuarios como responsables del área de redes sociales de un medio) esta técnica etnográfica permite saber de modo directo la opinión de los distintos actores del medio en internet (cibermedio) o del periodismo en red (ciberperiodismo). Por tanto, las entrevistas han de ser tanto cerradas como abiertas. Para las entrevistas mediadas por ordenador (CMC), pueden utilizarse los siguientes canales: correo electrónico, chat privado o incluso la propia red social, cuya característica interna disponga de un modelo de mensajería instantánea. Todo esto puede ser complementado con las entrevistas del modelo cara a cara (Face To Face o presencia física). Las entrevistas cara a cara han supuesto uno de los grandes retos de la investigación 
dado que los contactos físicos han permitido dotar de contexto offline a todos los datos que se hayan recogido de forma online, convirtiéndose en un complemento de la investigación con fuerza de cara a los resultados obtenidos.

\section{Investigación de archivos o Fuentes primarias}

La investigación de archivos es el análisis de materiales que se han almacenado para investigación, servicio y otros propósitos tanto oficiales como no oficiales (Angrosino, 2012: 74). Algunos etnógrafos prefieren llamarlos fuentes primarias. En el análisis de redes sociales, las fuentes primarias lo constituyen, en primer lugar, la bibliografía consultada que permite plantear el marco teórico y conceptual con los antecedentes de la investigación. Adicionalmente a la bibliografía, el investigador/a puede optar a presentar como fuente primaria el análisis de las publicaciones, tutoriales, aplicaciones propietarias (programas de software), guías o manuales de estilo asociadas a las redes sociales que las organizaciones de noticias puedan ya tener como la hoja de ruta a seguir en el desarrollo de los contenidos informativos.

Respecto a las técnicas cuantitativas, estas tienen como objetivo identificar tendencias en las conductas y pueden ser: encuestas, cuestionarios y censos. Una encuesta es un estudio en el cual el investigador obtiene los datos a partir de realizar un conjunto de preguntas normalizadas dirigidas a una muestra representativa o al conjunto total de la población estadística en estudio, formada a menudo por personas, empresas o entes institucionales, con el fin de conocer estados de opinión, características o hechos específicos. Las encuestas pueden ser:

- Encuestas cara a cara. Consisten en entrevistas directas y personales con cada encuestado que forman parte de la muestra seleccionada.

- Encuestas telefónicas: Este tipo de encuesta consiste en una entrevista vía telefónica con cada encuestado. También se basa en una muestra seleccionada.

- Encuestas por correo: Consiste en el envío de un cuestionario a los potenciales encuestados, pedirles que lo rellenen y hacer que lo devuelvan completado.

- Encuestas online: Este tipo de encuesta consiste en colocar un cuestionario en una página web o crear una encuesta en internet y enviarla a los correos electrónicos.

Para el estudio de las redes sociales, el tipo de encuesta que los investigadores suelen utilizar es de tipo Online. Consideran que el público al que va dirigida la encuesta está acostumbrado a interactuar con asiduidad en la red, por lo que el índice de respuestas puede ser más rápido y efectivo. Además de que muchos de los recursos de encuesta online, proporciona ayudas en la creación de tablas y gráficos para una posterior interpretación de los datos.

\section{Conclusiones}

El papel del investigador que utiliza las técnicas etnográficas en las redes sociales en Internet no sólo permite acercarse a una realidad para describirla de manera detallada, sino que requiere que el investigador se introduzca en esta interacción mediada por ordenador. A partir de ella, puede reflexionar sobre el uso directo del medio y el contexto en el que se efectúa. La etnografía virtual aplicada al estudio de las redes so- 
ciales permite además, utilizar su propia experiencia y combinarla con los elementos encontrados en el estudio y, que éstos a su vez, encuentren fundamento en las teorías existentes de antemano.

El camino que se ha de seguir en el estudio cualitativo de las redes sociales en Internet como parte de las audiencia digital de los medios para debe tener un fundamento etnográfico. La investigación del Periodismo en Internet (Ciberperiodismo) se abre a otras fuentes y al uso de técnicas de las ciencias sociales como la antropología y la etnografía. Este nuevo entorno de la investigación acrecienta el hecho de que la construcción del nuevo periodismo requiere de la imbricación de otras ciencias disciplinares lo que redundará en beneficio de las organizaciones de noticias, de la sociedad como parte activa en el proceso de construcción de la información, de los profesionales, de los académicos y de la propia disciplina del periodismo.

Se destaca que la intención de este artículo es mostrar una posible vía de trabajo. Por ello se exponen claramente los pasos seguidos y la metodología utilizada, quedando en evidencia la importancia de la participación directa del investigador. Consideramos que el proceso posterior es común a todas las investigaciones cualitativas sobre redes sociales virtuales como parte de la audiencia de los medios en Internet, dado que utilizan herramientas informáticas en su análisis.

Lo que en este artículo pretendemos indicar es como marcar la ruta etnográfica a utilizar como muestra de una vía eficaz de estudio y que aporta gran riqueza a la investigación de las comunicaciones mediada por ordenador.

\section{Referencias bibliográficas}

ANGROSINO, Michael (2012): Etnografía y observación participante en investigación cualitativa. Madrid, Ediciones Morata.

AUGÉ, Marc (2006): Hacia una antropología de los mundos contemporáneos. Barcelona. Gedisa.

CONTRERAS, Pau (2004): Me llamo Kohfam. Identidad hacker: una aproximación antropológica, Barcelona, Gedisa.

DOMÍNGUEZ, Daniel; BEAULIEU, Anne; ESTALELLA, Adolfo; GÓMEZ, Edgar; SCHNETTLER, Bernt; \& READ, Rosie (2007): "Etnografia virtual". Forum Qualitative Sozialforschung / Forum: Qualitative Social Research, 8 (3). [Consulta: 10 de enero de 2015]. En: http://www.qualitative-research.net/index.php/fqs/article/view/274/603

DUMIT, Joseph (2014) "Writing the Implosion: Teaching the World One Thing at a Time". Cultural Anthropology, 29, nº. 2, pp. 344-362. En: http://dx.doi.org /10.14506/ca29.2.09

ESCOBAR, Arturo (2005): "Bienvenidos a Cyberia. Notas para una antropología de la cibercultura" (Trad. Carlos Barragán). Revista de Estudios Sociales no 22, pp. 1535.

ETCHEVERS GOIJBERG, Nicole (2011): La comunicación emocional en Internet. Editorial Academia Española, Madrid. 
ETCHEVERS GOIJBERG, Nicole (2010): Ruta etnográfica para la comprensión de la comunicación on-line. [Consulta: 22 de noviembre de 2014]. Disponible en: http://ddd.uab.cat/pub/dim/16993748n1/16993748n1a2.pdf

FLORES, Jesús (Coord., 2014): Contenidos periodísticos en las redes sociales. Madrid. Fragua.

FLORES, Jesús (2009): "Nuevos modelos de comunicación, perfiles y tendencias en las redes sociales" [New Models of Communication, Profiles and Trends in Social Networks]. Comunicar, 33, pp. 73-81. (DOI: 10.3916/c33-2009-02-007).

HANNEMAN, Robert A. (2000): Introducción a los métodos del análisis de redes sociales. Departmento de Sociología de la Universidad de California Riverside, EE.UU. [Trad.: Maria Angela Petrizzo]

HINE, Christine (2004): Etnografia virtual. Barcelona, Editorial UOC.

MOLINA, José Luis y ÁVILA, Javier (2002): Antropología y redes sociales. Una introducción a Ucinet6-netdraw, Egonet y el análisis comparado con SPSS. UAB, Barcelona.

NADRUZ, Juan Andrés (2010): Las comunidades virtuales Xbox: interacción y socialización en el ciberespacio. [Consulta: 22 de noviembre de 2014]. Disponible en: http://www.unesco.org.uy/shs/fileadmin/templates/shs/archivos/anuario2009 /Nadruz.pdf

SÖREN ROMERO, André (2014): "Etnografía Virtual en redes sociales: el caso de Facebook", en $i S+D$ Fundación para la Investigación Social Avanzada. [Consulta: 20 de enero de 2015]. Disponible en: http://blog.isdfundacion.org/2014/12/01/etnografia-virtual-no-2-etnografia-virtual-en-redes-sociales-el-caso-de-facebookhei-man-2008/ 